\title{
DECOMPOSITION OF CURRENT THROUGH ACTIVE POWER FILTER FOR COMPENSATING HARMONICS CURRENT AND REACTIVE CURRENTS
}

\author{
V.Pardha Saradhi ${ }^{1}$, P.Dhana Selvi ${ }^{2}$ \\ ${ }^{1}$ Scholar M.Tech, EEE, AP,India,pardhume@gmail.com, ${ }^{2}$ Assistant professor, EEE, NBKRIST, AP, India
}

\begin{abstract}
APFs have now become an alternative solution to harmonic filtering technology. APF is to compensate the harmonic currents due to the non linear load.This paper mainly deals with shunt active power filter which has been widely used for harmonic elimination and supplying reactive currents. We can monitor the load continuously by using active power filter. The proposed scheme decomposes the load current into positive and negative sequence fundamental frequency active components, reactive component and harmonic components in modified $P Q$ theory. Estimating reference current signal nonlinear loads, especially power electronics converters based adjustable speed drives, computer power supplies, furnaces etc,by using PQ theory.
\end{abstract}

Index terms: Active power filter, harmonics, reactive currents, $P Q$ theory

\section{INTRODUCTION}

The Increased use of power electronic controlled equipment and non linear electronic devices in power system has given rise to type of voltage and current waveform distortion called as [1]'Harmonics'. The motive of electricity provider companies is to supply uninterrupted sinusoidal supply voltage and current of proper magnitude with negligible harmonic contents. However it is always difficult to achieve the above goals due to rapidly increasingly Active compensation or APF can solve the problem of harmonic and reactive power simultaneously.

Based on estimated reference current signal nonlinear loads, especially power electronics converters based adjustable speed drives, computer power supplies, furnaces, etc., generate harmonic currents and voltages in electrical systems. These harmonics cause equipment overheating, blown capacitor fuses, distorted voltage waveform, low power factor, eddy current losses on transformer, oscillatory torque response and reduces the efficiency of the system. To generate the compensating current, different approaches, such as notch filter, scalar control, instantaneous reactive power theory (IRPT) or modified PQ theory, synchronous detection method, SVPWM, direct power control etc., are used.

This paper proposes modified PQ theory for control strategy of active power filter. We can find the reference current by using modified PQ theory .A hysteresis -band PWM current controller is used to generate gating signal to the devices of the APF which is widely used due to its fast response. The reliability of proposed control scheme is verified by simulink model.

\section{SHUNT ACTIVE FILTERS}

A Shunt active filters have to be connected in parallel with the source .shunt active filters are by far the most widely accept and dominant filter of choice in most industrial processes. Fig: 1 show the system configuration of the shunt design.

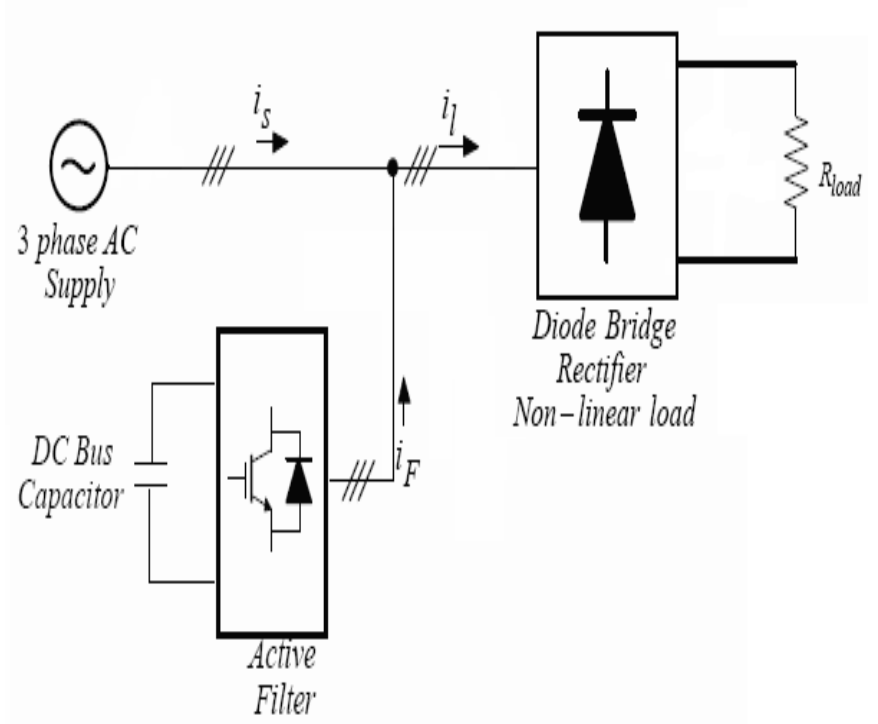

Fig-1: Schematic Diagram Of a System With Shunt Active Filter 
The objective of the shunt active filter is to supply opposing harmonic current to the nonlinear load effectively resulting in a net harmonic current. This means that the supply signals remain purely fundamental. Shunt filters also have the additional benefit of contributing to reactive power compensation and balancing of three -phase currents. Since the active filter is connected in parallel[2], only the compensation current plus a small amount of active fundamental current is carried in the unit. For an increased range of power ratings, several shunt active filters can be combined together to withstand higher currents.

The active filter is composed of a standard voltage source inverter bridge with a dc bus capacitor to provide an effective current control. The basic building blocks of the conventional parallel AF are shown in below fig. 1

For single-phase system there will be two legs of IGBT's and for three-phase system there will be three legs in case of three wire and four legs in case of four wire. The basic function of the proposed parallel $\mathrm{AF}$ is to eliminate harmonics and meet the reactive power requirements of the load locally so that the ac supply feeds only the sinusoidal balanced unity power factor currents.

The AF connected $\mathrm{n}$ shunt with the load, also enhances the system efficiency as the source does not process harmonic and reactive power. Here we have to generate the pulses to be supplied to the IGBT's by sending the reference source current and the actual source current to a PWM Generator. Then by switching the IGBT's by using these pulses ,Harmonics are eliminated.

\section{BASIS OF THE P-Q THEORY}

The instantaneous p-q theory is based on a set of instantaneous powers in the time domain. No restrictions are imposed on the voltage or current waveforms, and it can be applied threephase systems with or without neutral wire for three-phase generic voltage and current waveforms. Thus, it is valid not only in the steady state ,but also in the transient state. This theory is very efficient and flexible in designing controllers for power conditioners based on power electronic devices. The p$q$ theory first transforms voltages and currents from the abc to $\alpha \beta 0$ coordinates, and then defines instantaneous power on these coordinates. The p-q theory uses the $\alpha \beta 0$ transformations, also known as the Clarke transformation, which consists of real matrix that transforms three-phase voltages and currents into the $\alpha \beta 0$ stationary reference frames Therefore, the presentation of the $\mathrm{p}-\mathrm{q}$ theory will start with this transformation.

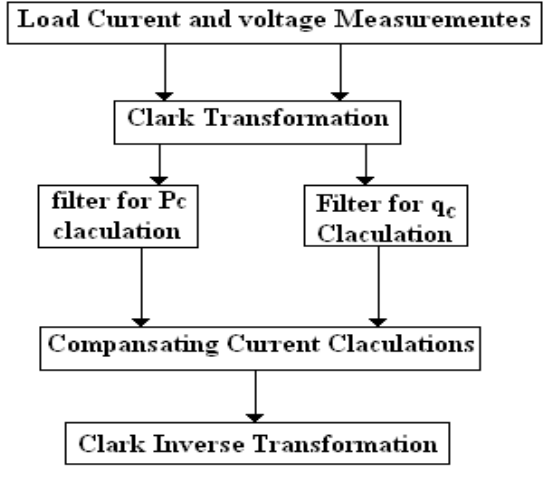

Fig 2: Flow chart for modified PQ theory

\subsection{The Clarke transformation}

The $\alpha \beta 0$ transformation (or) the Clarke transformation maps the three phase instantaneous voltages in the abc phases, Va, $\mathrm{Vb}, \mathrm{Vc}$, into the instantaneous voltages on the $\alpha \beta 0$-axes $\mathrm{V} \alpha, \mathrm{V} \beta, \mathrm{Vo}_{0}$.The clarke transformation and its inverse transformation of three phase generic voltages are given by

$\left[\begin{array}{l}v_{a} \\ v_{b} \\ v_{c}\end{array}\right]=\sqrt{\frac{2}{a}}\left[\begin{array}{ccc}1 / \sqrt{2} & 1 & 0 \\ 1 / \sqrt{2} & -1 / 2 & \sqrt{3} / 2 \\ 1 / \sqrt{2} & 1 / \sqrt{2} & -\sqrt{3} / 2\end{array}\right]\left[\begin{array}{l}v_{0} \\ v_{\alpha} \\ v_{\beta}\end{array}\right]$

Here va,vb,vc are(voltages at load) converted into vo,v $\alpha, v \beta$ by using above matrix.

$$
\left[\begin{array}{l}
i_{a} \\
i_{b} \\
i_{c}
\end{array}\right]=\sqrt{\frac{2}{3}}\left[\begin{array}{ccc}
1 / \sqrt{2} & 1 & 0 \\
1 / \sqrt{2} & -1 / 2 & \sqrt{3} / 2 \\
1 / \sqrt{2} & 1 / \sqrt{2} & -\sqrt{3} / 2
\end{array}\right]\left[\begin{array}{l}
i_{o} \\
i_{\alpha} \\
i_{\beta}
\end{array}\right]
$$

The advantage of the $\alpha \beta 0$ transformation is is to separate zero sequence currents from the abc transformation. The $\alpha$ and $\beta$ axes make no contribution to zero sequence components .No zero sequence component current exists in a three-phase, three wire system, so that io can be eliminated from the above equations thus resulting the simplification. If three -phase voltages are balanced in a four wire system, No zero-sequence voltage is presents that $\mathrm{V} 0$ can be eliminated. However, when Zero-sequence voltage and current components are present, the complete transformation has to be considered.

If Vo eliminated from the transformation matrices, the Clarke transformation and its inverse transformation becomes 
$\left[\begin{array}{l}v_{\alpha} \\ v_{\beta}\end{array}\right]=\sqrt{ } \frac{2}{a}\left[\begin{array}{ccc}1 & -1 / 2 & -1 / 2 \\ 0 & \sqrt{3} / 2 & -\sqrt{3} / 2\end{array}\right]\left[\begin{array}{l}v_{a} \\ v_{b} \\ v_{c}\end{array}\right]$

$\left[\begin{array}{l}v_{a} \\ v_{b} \\ v_{c}\end{array}\right]=\sqrt{\frac{2}{a}}\left[\begin{array}{cc}1 & 0 \\ -1 / 2 & \sqrt{3} / 2 \\ -1 / 2 & -\sqrt{3} / 2\end{array}\right]\left[\begin{array}{l}v_{\alpha} \\ v_{\beta}\end{array}\right]$

The above matrices suggest that an axis transformation as shown below. They are stationary axes and should not confused with the concepts of voltage and current phasors. Here instantaneous values of phase voltages and line currents are referred to the abc stationary axes are transformed into the $\alpha \beta$ stationary axes,(or) vice versa. The a,b,c, are spatially shifted for $2 \pi / 3$ rad from each other while the $\alpha$ and $\beta$ axis are orthogonal, and the $\alpha$-axis is parallel to the a axis.

\subsection{Calculation of Voltage and a Current Vectors When Zero Sequence Components Are Excluded}

If V0 can be neglected, an instantaneous voltage vector is defined from the instantaneous $\alpha$ and $\beta$ voltage components, that is,

$$
E=v \alpha+j v \beta
$$

Similarly,if io can be neglected the instantaneous current vector is defined as

$$
\mathrm{i}=\mathrm{i} \alpha+\mathrm{ji} \beta
$$

The above instantaneous vectors can be represented in a complex plane, where the real axis is the $\alpha$ axis, and the imaginary axis is the $\beta$ axis of the Clarke Transformation. It should be noted that the vectors defined above the functions of time, because they consists of the Clarke components of the instantaneous phase voltages and line currents in the three phase systems.

\subsection{The Instantaneous Powers of the p-q Theory}

The $\mathrm{p}-\mathrm{q}$ theory is defined in three phase systems with or without a neutral conductor. Three instantaneous powers-the instantaneous zero-sequence power po the instantaneous real power , and the instantaneous imaginary power q-are defined from the instantaneous phase voltages and line currents on the $\alpha \beta$ axes as

$$
\left[\begin{array}{c}
p_{0} \\
\hdashline p \\
q
\end{array}\right]=\left[\begin{array}{c:cc}
v_{0} & 0 & 0 \\
\hdashline 0 & v_{\alpha} & v_{\beta} \\
0 & v_{\beta} & -v_{\alpha}
\end{array}\right]\left[\begin{array}{c}
i_{0} \\
\hdashline i_{\alpha} \\
i_{\beta}
\end{array}\right]
$$

There are no zero-sequence current components in three phase , three wire systems, tht is, is $=0$. In this cae, only the instantaneous powers defined on the $\alpha \beta$ axe exist, because the product voio is always is zero .Hence, in three-phase, three wire systems, the instantaneous real power $\mathrm{p}$ represents the total energy flow per unity in terms of $\alpha \beta$ components.

\subsection{The p-q theory in three-phase, three-wire systems}

The instantaneous complex power ' $\mathrm{s}$ ' is defined as the product of voltage vector e and the conjugate of the current $i^{*}$,given in the form of complex numbers.

$$
E i^{*}=(v \alpha+j v \beta)(i \alpha-i \beta)
$$

$\left[\begin{array}{l}p_{1} \\ q\end{array}\right]=\left[\begin{array}{ll}v_{\alpha} & w_{\beta} \\ -v_{\beta} & w_{\alpha}\end{array}\right]\left[\begin{array}{l}i_{\alpha} \\ i_{\beta}\end{array}\right]$

Instantaneous active current on the $\alpha$ axis

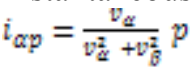

Instantaneous reactive current on the $\alpha$ axis $i_{\propto q}=\frac{w_{\alpha}}{w_{\alpha}^{2}+w_{g}^{2} q}$

Instantaneous active current on the $\beta$ axis $i_{\beta q}=\frac{-w_{b}}{w_{\alpha}^{2}+D_{0}^{2} q} q$

Instantaneous reactive current on the $\beta$ axis $i_{\beta \alpha}=\frac{w_{\theta}}{w_{\alpha}^{2}+D_{\theta}^{2} p} p$

Only we have consider active currents

$\left[\begin{array}{l}i_{a} \\ i_{b} \\ i_{c}\end{array}\right]=\left[\begin{array}{cc}1 & 0 \\ -\frac{1}{2} & \frac{\sqrt{3}}{2} \\ -\frac{1}{2} & -\frac{\sqrt{3}}{2}\end{array}\right]\left[\begin{array}{l}i_{\alpha p} \\ i_{\beta p}\end{array}\right]=i_{\text {aberef }}$

Iref=iabc-iabcref 


\section{MATLAB-BASED SIMULATION AND}

\section{RESULTS}

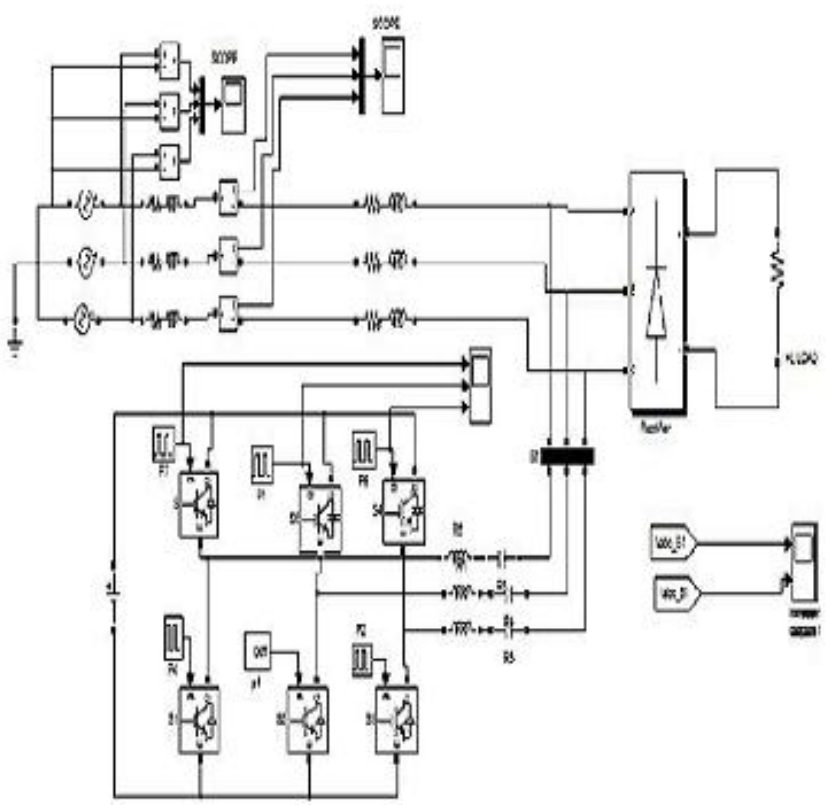

Fig 3: simulation diagram for APF with Nonlinear load

The power source, rectifier fed equivalent resistive load with input side inductive filter, three phase resistive load, APF are modeled in MATLAB using Power System Blockset. Fig. 2 depicts the setup used to estimate the performance of the APF with proposed control scheme through simulation. The source block consists of a three-phase voltage source "infinite bus" with series impedance representing the relevant short-circuit impedance of the supplying grid to gauge the performance of the APF with proposed scheme under specified conditions. The considered loads to evaluate the effectiveness of the proposed scheme are diode rectifier with inductive filter on source side and star connected resistive $(\mathrm{R})$ load connected to ac mains.

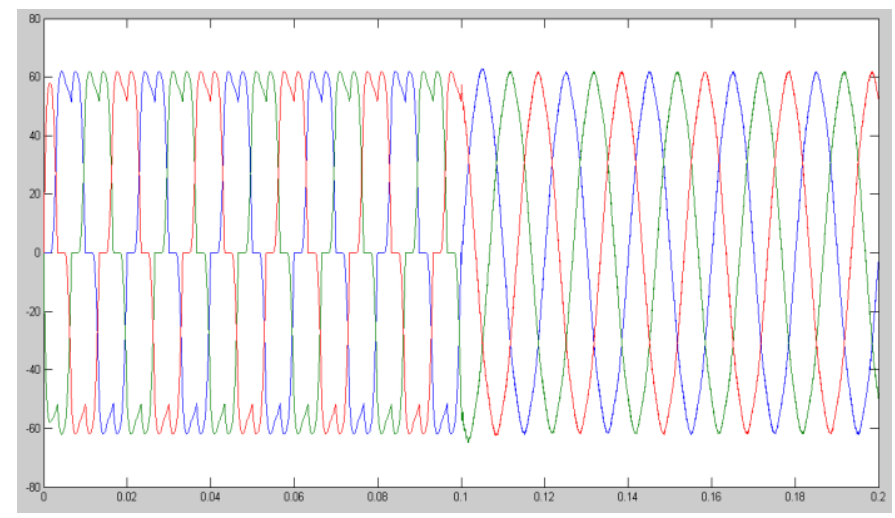

Fig 4: Source current for Nonlinear load

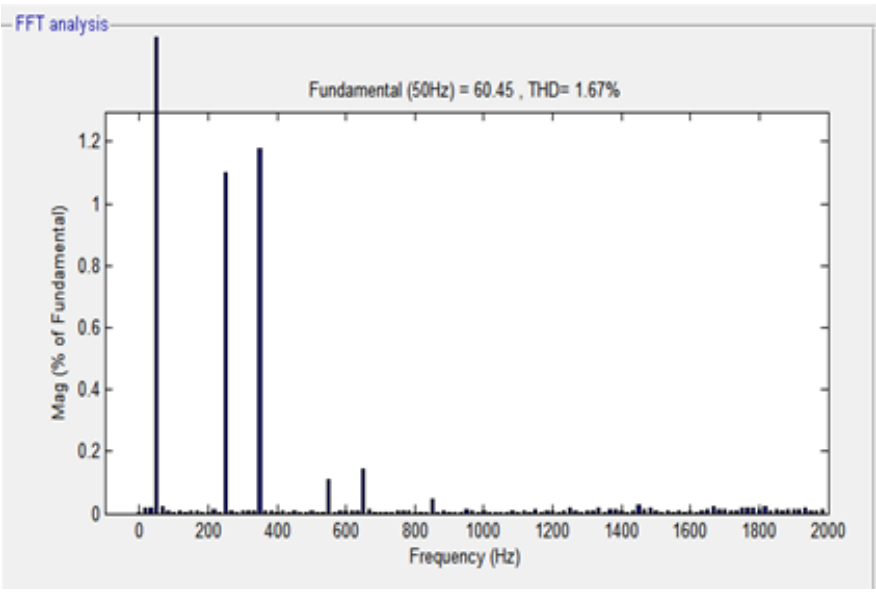

Fig 5:FFT Analysis for Nonlinear load

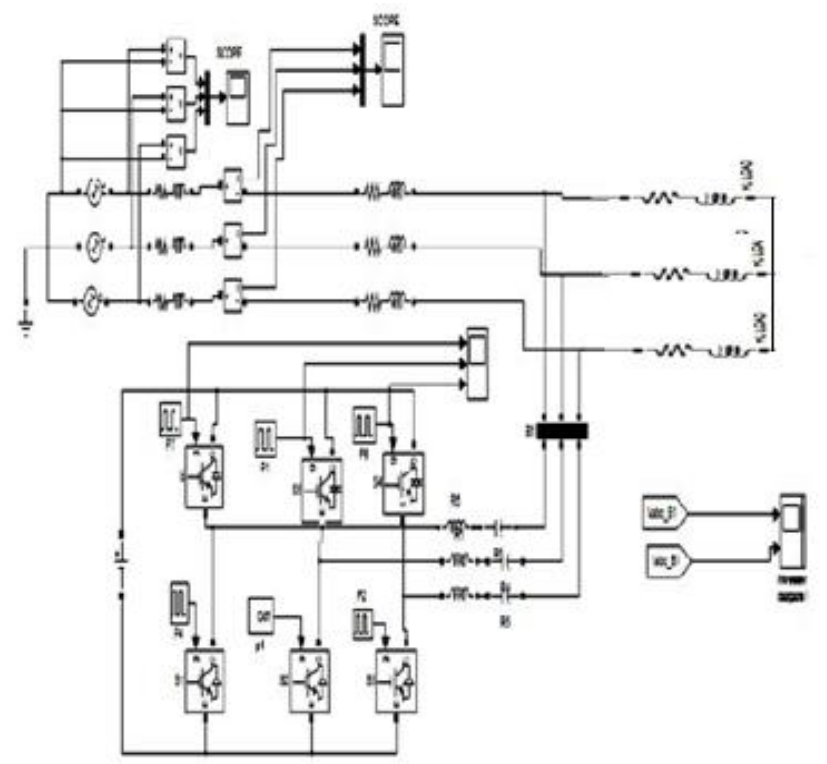

Fig 6: simulation diagram unbalanced RL load

In this simulation results at $0.1 \mathrm{sec}$ filter activated before filter not activate. We can activate filter at any time in the simulation by connecting timer.

The RL unbalanced load, the APF supply reactive current to the load. So reduce the burden on the source. 


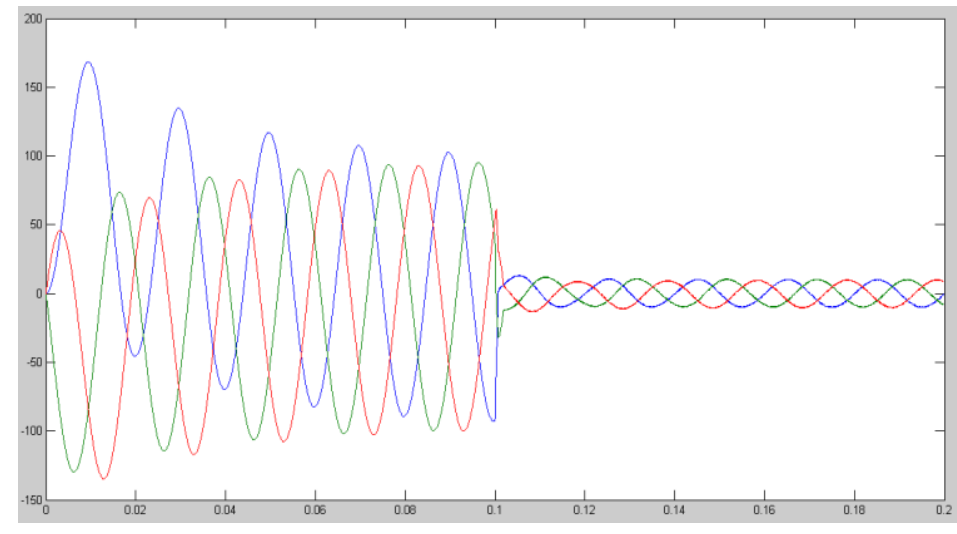

Fig 6: source current for Unbalanced RL load

This filter activated at $0.1 \mathrm{sec}$, from this instant APF supply the reactive current. This filter we can use for RLE loads.

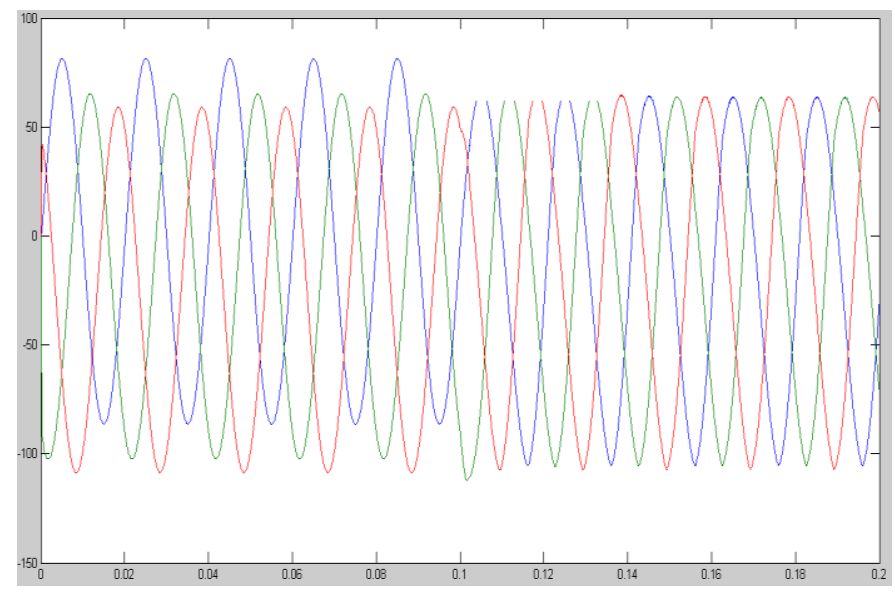

Fig 7: Source current for the RLE load

\section{CONCLUSIONS}

A current decomposition technique based on instantaneous power theory for shunt active power filters is studied, a simulink model is designed and total harmonic distortion is calculated using FFT analysis. Active power filter which has been used here monitors the load current constantly and continuously adapt to the changes in load harmonics. The performance of three phase shunt active power filter using instantaneous power theory

\section{REFERENCES}

[1] Schlabbach, D. Blume, and T. Stephanblome, "Voltage Quality in Electrical Power Systems", ser. PEE Series. New York: IEE Press,2001.

[2] L. Gyugyi and E. C. Strycula, "Active AC power filter," in Proc. IEEE IAS Annu. Meeting, 1976, pp. 529-529.

[3] H. Akagi, Y. Kanazawa, and A. abae, "Generalized theory of the instantaneous reactive power in three- phase circuits,"in Proc. IEEE and JIEE IPEC, 1983, pp. 821-827.

[4] Y. Komatsu and T. Kawabata, "Experimental comparison of pq and extended pq method for active power filter," in Proc. EPE, 1997, pp.2.729-2.734

[5] V. Soares, P. Verdelho, and P. D. Marques, "Active power filter control circuit based on instantaneous active and reactive current id - iq method," in Proc. IEEE PESC, 1997, pp. 106-101.

[6] B. Singh, K. Al-Haddad, and A. Chandra, "A new control approach to three-phase active filter for harmonics and reactive power compensation,"

[7] IEEE Trans. Power Syst., vol. 13, no. 1, pp. 133-138, Feb. 1998. "Active power filters for non-linear loads".Instantaneous Power Theory Based Active Power Filter: A Mat lab/ Simulink Approach.

[8] Generalized theory of the instantaneous reactive power in three-phase circuits," in Proc. IEEE and JIEE IPEC, 1983, pp. 821-827.

[9] Harashima F., Inaba H. and Tsuboi K., “A close-loop control system for the reduction of reactive power required by electronic converters,"Toshiba Electric Co., Tokyo, Japan, Oct. 1975.

[10] Knoell H., "3 kW-switch-mode supply providing Sinusoidal mains current and large range of dc-output," PCI, Munich, Germany, 1980.

[11] Nastran J.. "Active Power Filter," dissertation presented at the Faculty of Electrical Engineering, Ljubljana, Yugoslavia, 1985. 